\title{
Synthesis and Antifungal Activity of a Novel Series of Alkyldimethylamine Cyanoboranes and Their Derivatives
}

\author{
Khuloud Takrouri ${ }^{1}$, Gal Oren ${ }^{2}$, Itzhack Polacheck ${ }^{2}$, Edward Sionov ${ }^{2}$, Eli Shalom ${ }^{1}$, \\ Jehoshua Katzhendler ${ }^{1}$ and Morris Srebnik ${ }^{1 *}$. \\ ${ }^{1}$ Department of Natural Products and Medicinal Chemistry, School of Pharmacy, \\ Hebrew University of Jerusalem, 91120 Israel. \\ ${ }^{2}$ Department of Clinical Microbiology and Infectious Diseases, Hadassah-Hebrew \\ University Medical Center.
}

\section{Supporting Information}

\section{Spectroscopic analysis:}

All the new compounds were fully characterized by ${ }^{1} \mathrm{H},{ }^{11} \mathrm{~B}$, and ${ }^{13} \mathrm{C}$ NMR, FT-IR, and elemental analysis. For the alkyldimethylamine cyanoboranes 8-9, 14-18, and 28, and the diamine bis-cyanoboranes $\mathbf{1 9 - 2 4}$, triplets due to the $\mathrm{N}_{-} \mathrm{CH}_{2} \mathrm{R}$ group in the ${ }^{1} \mathrm{H}$ NMR spectra and singlets in the ${ }^{13} \mathrm{C}$ NMR spectra were observed. An expected downfield shift of the methyl groups attached to the nitrogen atom in comparison to the starting trimethylamine cyanoborane was obtained. For the $\beta$-hydroxyamine cyanoboranes, $\mathbf{1 3 , 1 5}$ and 26, multiplets due to $\mathrm{H}-\mathrm{C}-\mathrm{OH}$ appeared downfield between (4.07-6.01 ppm) in the ${ }^{1} \mathrm{H}$ NMR spectra, and between (53.01-77.60 ppm) in ${ }^{13} \mathrm{C}$ NMR spectra. The methylene hydrogens $\left(\mathrm{N}-\mathrm{CH}_{2} \mathrm{CR}(\mathrm{OH}) \mathrm{R}^{\prime}\right)$ of the $\beta$-hydroxyamine cyanoboranes $\mathbf{1 3 , 1 5}$ and $\mathbf{2 6}$, and the $\beta$-alkoxyamine cyanoborane 7 , are diastereotopic and in most cases showed different peaks for each hydrogen. The $\mathrm{N}$-methyl groups $\left(\mathrm{NMe}_{2}\right)$ for these derivatives are also diastereotopic and peaks for each group were evident in both the in ${ }^{1} \mathrm{H}$ and ${ }^{13} \mathrm{C}$ NMR spectra (see chemical experimental section). In the ${ }^{11} \mathrm{~B}$ NMR triplets were obtained for all the amine cyanoboranes structure types prepared due to B-H coupling. 
The ${ }^{1} \mathrm{H}$ and ${ }^{13} \mathrm{C}$ NMR spectra for the bromo derivatives, 10-11, 20-21, showed an expected downfield shifts of the $\mathrm{CH}_{2}-\mathrm{N}$ and $\mathrm{CH}_{3}-\mathrm{N}$ peaks due to the replacement of hydrogen atom by bromine atom (see chemical experimental section). In the ${ }^{11} \mathrm{~B}$, similar downfield shifts were observed, and the multiplicity due to $\mathrm{BH}$ coupling changed from triplets for $\mathrm{BH}_{2}$ in the un-brominated amine cyanoboranes, to doublets and singlets for the monobromo and dibromo derivatives respectively. Diasteriotopic peaks for the carbon atom of the $\mathrm{N}-\mathrm{CH}_{2}$ fragment appeared in all ${ }^{13} \mathrm{C}$ spectra for the chiral boron atom containing compounds $\mathbf{1 0}$ and 20 (see chemical experimental section). In the dibromo derivatives $\mathbf{1 1}$ and $\mathbf{2 1}$, the ${ }^{1} \mathrm{H}$ and ${ }^{13} \mathrm{C}$ NMR spectra showed an expected upfield shift of the $\mathrm{CH}_{2}-\mathrm{N}$ and $\mathrm{CH}_{3}-\mathrm{N}$ peaks due to the absence of the electronegative bromine atom. In the ${ }^{11} \mathrm{~B}$, similar upfield shifts were observed, but were still downfiled in comparison to the ${ }^{11} \mathrm{~B}$ chemical shifts of the monomers of the corresponding amine cyanoboranes $\left(\mathrm{A}: \mathrm{BH}_{2} \mathrm{CN}\right)$. In addition, the triplets caused by $\mathrm{BH}$ coupling in $\mathrm{BH}_{2}$ fragment was replaced by doublets caused by the $\mathrm{BH}$ coupling in the BH-BH fragment. In the flouro- derivative, i.e. compound 12, the ${ }^{1} \mathrm{H}$ and ${ }^{13} \mathrm{C}$ NMR spectra showed an upfield shift of the $\mathrm{Me}_{2} \mathrm{~N}$ peak when bromine was replaced by the highly electronegative fluorine. The ${ }^{11}$ B-NMR peaks were obtained as a doublet of doublets due to splitting by both hydrogen and fluorine atoms attached to boron. In the ${ }^{19} \mathrm{~F}$ NMR, a quartet of doublets was observed due to the splitting of both boron and hydrogen neighboring atoms.

\section{Analytical data:}

Dimethylnonylamine cyanoborane (8). White solid, $84 \%(0.177 \mathrm{~g})$ yield. ${ }^{1} \mathrm{H}$ NMR $\left(\mathrm{CDCl}_{3}\right): \delta 0.86(\mathrm{t}, 3 \mathrm{H}, J=7.2 \mathrm{~Hz}), 1.29($ broad s, 10H), 1.63 (hept, 2H, $J=3.6), 1.80$ (pent, $2 \mathrm{H}, J=7.2), 2.65(\mathrm{~s}, 6 \mathrm{H}), 2.83(\mathrm{t}, 2 \mathrm{H}, J=5.0)$, (HB cannot be detected $).{ }^{13} \mathrm{C}\left\{{ }^{1} \mathrm{H}\right\} \mathrm{NMR}$ $\left(\mathrm{CDCl}_{3}\right): \delta 14.13,22.81,23.23,27.13,29.44,29.65,32.09,45.18,50.03,63.84,(\mathrm{CB}$ cannot 
be detected). ${ }^{11} \mathrm{~B}$ NMR $\left(\mathrm{CDCl}_{3}\right): \delta-16.01\left(\mathrm{t}, J_{\mathrm{B}-\mathrm{H}}=97.1 \mathrm{~Hz}\right) . \mathrm{IR}\left(\mathrm{KBr}, \mathrm{cm}^{-1}\right): 2920(\mathrm{~B}-\mathrm{H})$, $2850(\mathrm{C}-\mathrm{H}), 2358(\mathrm{C} \equiv \mathrm{N}), 1469(\mathrm{C}-\mathrm{N}), 432(\mathrm{~B}-\mathrm{N})$.

1-(Dimethylamino)-undecan-2-ol cyanoborane (13). Yellow oil, $84 \%(0.214 \mathrm{~g})$ yield. ${ }^{1} \mathrm{H}$ $\operatorname{NMR}\left(\mathrm{CDCl}_{3}\right): \delta 0.86(\mathrm{t}, 3 \mathrm{H}, J=6.3 \mathrm{~Hz}), 1.25($ broad s, $14 \mathrm{H}), 1.43(\mathrm{~m}, 2 \mathrm{H}), 2.75(\mathrm{~s}, 3 \mathrm{H})$, $2.78(\mathrm{~s}, 3 \mathrm{H}), 2.89(\mathrm{~m}, 2 \mathrm{H}), 4.18(\mathrm{~m}, 1 \mathrm{H}),(\mathrm{HB}$ cannot be detected $) .{ }^{13} \mathrm{C}\left\{{ }^{1} \mathrm{H}\right\} \mathrm{NMR}\left(\mathrm{CDCl}_{3}\right)$ : $\delta 14.33,22.89,25.35,29.54,29.73,29.79,32.11,36.87,43.61,45.17,46.99,53.01,(\mathrm{CB}$ cannot be detected). ${ }^{11} \mathrm{~B}$ NMR $\left(\mathrm{CDCl}_{3}\right): \delta-\delta-16.67\left(\mathrm{t}, J_{\mathrm{B}-\mathrm{H}}=97.1 \mathrm{~Hz}\right)$. IR (neat, $\left.\mathrm{cm}^{-1}\right)$ : 3447 (O-H), 2926 (B-H), $2954(\mathrm{C}-\mathrm{H}), 2334(\mathrm{C} \equiv \mathrm{N}), 1543(\mathrm{C}-\mathrm{N}), 443(\mathrm{~B}-\mathrm{N})$.

Dodecyldimethylamine cyanoborane (14). Yellow oil, $87 \%\left(0.219\right.$ g) yield. ${ }^{1} \mathrm{H}$ NMR $\left(\mathrm{CDCl}_{3}\right): \delta 0.84(\mathrm{t}, 3 \mathrm{H}, J=7.2 \mathrm{~Hz}), 1.22($ broad s, 16H), 1.64 (hept, $2 \mathrm{H}, J=3.6), 1.80$ (pent, $2 \mathrm{H}, J=7.2), 2.60(\mathrm{~s}, 6 \mathrm{H}), 2.80(\mathrm{t}, 2 \mathrm{H}, J=5.0)$, (HB cannot be detected). ${ }^{13} \mathrm{C}\left\{{ }^{1} \mathrm{H}\right\} \mathrm{NMR}$ $\left(\mathrm{CDCl}_{3}\right): \delta 14.30,22.87,23.47,27.11,28.36,28.96,29.41,29.64,29.68,29.77,32.09$, 33.03, 34.22, 50.00, 63.78, $\left(\mathrm{CB}\right.$ cannot be detected). ${ }^{11} \mathrm{~B}$ NMR $\left(\mathrm{CDCl}_{3}\right): \delta-14.80\left(\mathrm{t}, J_{\mathrm{B}-\mathrm{H}}=\right.$ 102.6 Hz). IR (KBr, cm $\left.{ }^{-1}\right)$ : $2921(\mathrm{~B}-\mathrm{H}), 2854(\mathrm{C}-\mathrm{H}), 2361(\mathrm{C} \equiv \mathrm{N}), 1541(\mathrm{C}-\mathrm{N}), 440(\mathrm{~B}-\mathrm{N})$.

1-(dimethylamino)-dodecan-2-ol cyanoborane (15). Yellow oil, $85 \%(0.228 \mathrm{~g})$ yield. ${ }^{1} \mathrm{H}$ $\operatorname{NMR}\left(\mathrm{CDCl}_{3}\right): \delta 0.80(\mathrm{t}, 3 \mathrm{H}, J=7.2 \mathrm{~Hz}), 1.18($ broad s, 14H), $1.45(\mathrm{~m}, 4 \mathrm{H}), 2.67(\mathrm{~s}, 3 \mathrm{H})$, $2.72(\mathrm{~s}, 3 \mathrm{H}), 2.85(\mathrm{~m}, 2 \mathrm{H}), 4.07(\mathrm{~m}, 1 \mathrm{H}),(\mathrm{HB}$ cannot be detected $) .{ }^{13} \mathrm{C}\left\{{ }^{1} \mathrm{H}\right\} \mathrm{NMR}\left(\mathrm{CDCl}_{3}\right)$ : $\delta 14.26,22.83,25.33,29.49,29.76,31.05,32.06,36.84,43.60,49.30,52.49,52.89,68.59$, (CB cannot be detected). ${ }^{11} \mathrm{~B}$ NMR $\left(\mathrm{CDCl}_{3}\right): \delta-14.87\left(\mathrm{t}, J_{\mathrm{B}-\mathrm{H}}=102.7 \mathrm{~Hz}\right) . \mathrm{IR}\left(\mathrm{KBr}, \mathrm{cm}^{-1}\right)$ : $2920(\mathrm{~B}-\mathrm{H}), 2854(\mathrm{C}-\mathrm{H}), 2360(\mathrm{C} \equiv \mathrm{N}), 1464(\mathrm{C}-\mathrm{N}), 430(\mathrm{~B}-\mathrm{N})$. 
Dimethyltridecylamine cyanoborane (16). White solid, $84 \%(0.224 \mathrm{~g})$ yield. ${ }^{1} \mathrm{H}$ NMR $\left(\mathrm{CDCl}_{3}\right): \delta 0.87(\mathrm{t}, 3 \mathrm{H}, J=7.2 \mathrm{~Hz}), 1.25($ broad s, $16 \mathrm{H}), 1.41(\mathrm{~m}, 2 \mathrm{H}), 1.65$ (hept, $2 \mathrm{H}, J=$ 3.6), 1.84 (pent, $2 \mathrm{H}, J=7.2), 2.63(\mathrm{~s}, 6 \mathrm{H}), 2.84$ (t, 2H, $J=3.9$ ), (HB cannot be detected). ${ }^{13} \mathrm{C}$ $\left\{{ }^{1} \mathrm{H}\right\}$ NMR $\left(\mathrm{CDCl}_{3}\right): \delta 14.35,22.91,23.49,27.14,28.40,29.00,29.57,29.67,29.84,32.13$, 33.06, 34.31, 50.02, 64.00, $\left(\mathrm{CB}\right.$ cannot be detected). ${ }^{11} \mathrm{~B} \mathrm{NMR}\left(\mathrm{CDCl}_{3}\right): \delta-15.55\left(\mathrm{t}, J_{\mathrm{B}-\mathrm{H}}=\right.$ 106.3 Hz). IR (KBr, cm $\left.{ }^{-1}\right): 2921(\mathrm{~B}-\mathrm{H}), 2852(\mathrm{C}-\mathrm{H}), 2345(\mathrm{C} \equiv \mathrm{N}), 1542(\mathrm{C}-\mathrm{N}), 440(\mathrm{~B}-\mathrm{N})$.

Dimethylpentadecylamine cyanoborane (17). White solid, $86 \%(0.253 \mathrm{~g})$ yield. ${ }^{1} \mathrm{H}$ NMR $\left(\mathrm{CDCl}_{3}\right): \delta 0.87(\mathrm{t}, 3 \mathrm{H}, J=7.2 \mathrm{~Hz}), 1.25($ broad s, 20H), $1.41(\mathrm{~m}, 2 \mathrm{H}), 1.66$ (hept, $2 \mathrm{H}, J=$ 3.6), 1.84 (pent, $2 \mathrm{H}, J=7.2), 2.63(\mathrm{~s}, 6 \mathrm{H}), 2.84$ (t, 2H, $J=3.6$ ), (HB cannot be detected). ${ }^{13} \mathrm{C}$ $\left\{{ }^{1} \mathrm{H}\right\} \mathrm{NMR}\left(\mathrm{CDCl}_{3}\right): \delta 14.35,22.92,23.50,27.16,28.41,29.01,29.60,29.68,29.78,29.89$, 32.16, 33.08, 34.27, 47.04, 50.02, 63.84, (CB cannot be detected). ${ }^{11} \mathrm{~B}$ NMR $\left(\mathrm{CDCl}_{3}\right): \delta$ $15.89\left(\mathrm{t}, J_{\mathrm{B}-\mathrm{H}}=106.3 \mathrm{~Hz}\right) . \mathrm{IR}\left(\mathrm{KBr}, \mathrm{cm}^{-1}\right): 2919(\mathrm{~B}-\mathrm{H}), 2852(\mathrm{C}-\mathrm{H}), 2346(\mathrm{C} \equiv \mathrm{N}), 1538(\mathrm{C}-$ N), $440(\mathrm{~B}-\mathrm{N})$.

Heptadecyldimethylamine cyanoborane (18). White solid, $83 \%\left(0.268\right.$ g) yield. ${ }^{1} \mathrm{H}$ $\operatorname{NMR}\left(\mathrm{CDCl}_{3}\right): \delta 0.88(\mathrm{t}, 3 \mathrm{H}, J=7.2 \mathrm{~Hz}), 1.25(\operatorname{broad} \mathrm{s}, 24 \mathrm{H}), 1.41(\mathrm{~m}, 2 \mathrm{H}), 1.65(\mathrm{~m}, 2 \mathrm{H})$, 1.85 (pent, 2H, J= 7.2), $2.64(\mathrm{~s}, 6 \mathrm{H}), 2.85(\mathrm{t}, 2 \mathrm{H}, J=4.2)$, (HB cannot be detected). ${ }^{13} \mathrm{C}$ $\left\{{ }^{1} \mathrm{H}\right\}$ NMR $\left(\mathrm{CDCl}_{3}\right): \delta 14.36,22.93,28.42,29.01,29.60,29.91,23.16,33.07,34.33,48.25$, 52.10, 62.95, (CB cannot be detected). ${ }^{11} \mathrm{~B}$ NMR $\left(\mathrm{CDCl}_{3}\right): \delta-16.04\left(\mathrm{t}, J_{\mathrm{B}-\mathrm{H}}=106.3 \mathrm{~Hz}\right) . \mathrm{IR}$ $\left(\mathrm{KBr}, \mathrm{cm}^{-1}\right)$ : $2921(\mathrm{~B}-\mathrm{H}), 2852(\mathrm{C}-\mathrm{H}), 2344(\mathrm{C} \equiv \mathrm{N}), 1542(\mathrm{C}-\mathrm{N}), 433(\mathrm{~B}-\mathrm{N})$.

$\mathbf{N}, \mathbf{N}, \mathbf{N}^{\prime}, \mathbf{N}^{\prime}$-Tetramethyldodecane-1,12-diamine bis-cyanoborane (23). Yellowish solid, $88 \%(0.294 \mathrm{~g})$ yield. ${ }^{1} \mathrm{H}$ NMR $\left(\mathrm{CDCl}_{3}\right): \delta 1.27$ (broad s, $\left.12 \mathrm{H}\right), 1.66(\mathrm{~m}, 4 \mathrm{H}), 1.98(\mathrm{~m}, 4 \mathrm{H})$, 
$2.63(\mathrm{~s}, 12 \mathrm{H}), 2.85(\mathrm{t}, 4 \mathrm{H}, J=3.9 \mathrm{~Hz}),\left(\mathrm{HB}\right.$ cannot be detected) ${ }^{13} \mathrm{C}\left\{{ }^{1} \mathrm{H}\right\} \mathrm{NMR}\left(\mathrm{CDCl}_{3}\right)$ : $\delta 23.45,27.06,29.33,29.53,29.86,50.07,63.77$, (CB cannot be detected). ${ }^{11} \mathrm{~B}$ NMR $\left(\mathrm{CDCl}_{3}\right): \delta-15.53\left(\mathrm{t}, J_{\mathrm{B}-\mathrm{H}}=106.3 \mathrm{~Hz}\right) . \mathrm{IR}\left(\mathrm{KBr}, \mathrm{cm}^{-1}\right): 2921(\mathrm{~B}-\mathrm{H}), 2852(\mathrm{C}-\mathrm{H}), 2362$ $(\mathrm{C} \equiv \mathrm{N}), 1542(\mathrm{C}-\mathrm{N}), 440(\mathrm{~B}-\mathrm{N})$.

$\mathbf{N}, \mathbf{N}, \mathbf{N}^{\prime}, \mathbf{N}^{\prime}$-Tetramethyltetradecane-1,14-diamine bis-cyanoborane (24). White solid, 87 $\%\left(0.315\right.$ g) yield. ${ }^{1} \mathrm{H}$ NMR $\left(\mathrm{CDCl}_{3}\right): \delta 1.22$ (broad s, $\left.16 \mathrm{H}\right), 1.62(\mathrm{~m}, 4 \mathrm{H}), 1.81(\mathrm{~m}, 4 \mathrm{H})$, $2.60(\mathrm{~s}, 12 \mathrm{H}), 2.80(\mathrm{~m}, 4 \mathrm{H}),\left(\mathrm{HB}\right.$ cannot be detected) ${ }^{13} \mathrm{C}\left\{{ }^{1} \mathrm{H}\right\} \mathrm{NMR}\left(\mathrm{CDCl}_{3}\right): \delta 23.49$, 27.11, 28.96, 29.62, 33.03, 34.34, 50.07, 63.82, (CB cannot be detected). ${ }^{11} \mathrm{~B}$ NMR $\left(\mathrm{CDCl}_{3}\right): \delta-14.86\left(\mathrm{t}, J_{\mathrm{B}-\mathrm{H}}=112.3 \mathrm{~Hz}\right)$. IR $\left(\mathrm{KBr}, \mathrm{cm}^{-1}\right): 2920(\mathrm{~B}-\mathrm{H}), 2853(\mathrm{C}-\mathrm{H}), 2334$ $(\mathrm{C} \equiv \mathrm{N}), 1542(\mathrm{C}-\mathrm{N}), 440(\mathrm{~B}-\mathrm{N})$.

Table of elemental analysis:

\begin{tabular}{|c|c|c|c|c|c|c|c|}
\hline \multirow{2}{*}{ Compound } & \multirow{2}{*}{ Formula } & \multicolumn{3}{|c|}{ Calculated } & \multicolumn{3}{|c|}{ Found } \\
\hline & & $\mathrm{C} \%$ & H\% & $\mathrm{N \%}$ & $\mathrm{C \%}$ & H\% & N\% \\
\hline 8 & $\mathrm{C}_{12} \mathrm{H}_{27} \mathrm{BN}_{2}$ & 68.58 & 12.95 & 13.33 & 68.56 & 13.30 & 13.35 \\
\hline 13 & $\mathrm{C}_{14} \mathrm{H}_{31} \mathrm{BN}_{2} \mathrm{O}$ & 66.14 & 12.29 & 11.02 & 66.11 & 12.21 & 11.10 \\
\hline 14 & $\mathrm{C}_{15} \mathrm{H}_{33} \mathrm{BN}_{2}$ & 71.42 & 13.19 & 11.11 & 71.40 & 13.11 & 11.09 \\
\hline 15 & $\mathrm{C}_{15} \mathrm{H}_{33} \mathrm{BN}_{2} \mathrm{O}$ & 67.16 & 12.40 & 10.44 & 67.14 & 12.41 & 10.49 \\
\hline 16 & $\mathrm{C}_{15} \mathrm{H}_{33} \mathrm{BN}_{2}$ & 72.17 & 13.25 & 10.52 & 72.15 & 13.21 & 10.49 \\
\hline 17 & $\mathrm{C}_{18} \mathrm{H}_{39} \mathrm{BN}_{2}$ & 73.45 & 13.36 & 9.52 & 73.43 & 13.38 & 9.49 \\
\hline 18 & $\mathrm{C}_{20} \mathrm{H}_{43} \mathrm{BN}_{2}$ & 74.51 & 13.44 & 8.69 & 74.48 & 13.40 & 8.63 \\
\hline 23 & $\mathrm{C}_{18} \mathrm{H}_{40} \mathrm{~B}_{2} \mathrm{~N}_{4}$ & 64.70 & 12.07 & 16.77 & 64.73 & 12.08 & 16.73 \\
\hline 24 & $\mathrm{C}_{20} \mathrm{H}_{44} \mathrm{~B}_{2} \mathrm{~N}_{4}$ & 66.32 & 12.24 & 15.47 & 66.30 & 12.21 & 15.52 \\
\hline
\end{tabular}

